\title{
Spontaneous Rupture of Multiple Intrahepatic Cholangiocarcinoma in a Pregnant Patient
}

\author{
Min Hee Lee', Hae Kyung Lee', Boem Ha Yi', Seo-Youn Choi' , Hee Kyung Kim² , Jun Chul Chung ${ }^{3}$ \\ Departments of ${ }^{1}$ Radiology, ${ }^{2}$ Pathology, and ${ }^{3}$ Surgery, Soonchunhyang University Bucheon Hospital, Bucheon, Korea
}

\begin{abstract}
Spontaneous rupture of intrahepatic cholangiocarcinoma is rare due to its abundant fibrous stroma. We report our experience in a case of spontaneous rupture of intrahepatic cholangiocarcinoma in a pregnant patient. In our case, multiple hepatic masses with intratumoral hemorrhage were noted on post-delivery computed tomography and magnetic resonance imaging and they caused hemoperitoneum. A right hemi-hepatectomy was carried out to control the bleeding and the diagnosis of intrahepatic cholangiocarcinoma with metastasis was performed.
\end{abstract}

Keywords: Cholangiocarcinoma; Intrahepatic bile ducts; Rupture; Pregnancy

\section{INTRODUCTION}

Spontaneous rupture of a hepatic tumor is a life threatening condition. Hepatocellular carcinoma (HCC), hepatic hemangioma, hepatic adenoma, and metastasis may rupture [1]. But rupture of cholangiocarcinoma is rarely reported. Herein, we report a patient with spontaneously ruptured intrahepatic cholangiocarcinoma which was presented during her pregnancy.

\section{CASE REPORT}

A 32-year-old pregnant woman at 35 weeks and 2 days of gestation presented with right sided abdominal pain. On examination, her vital signs were stable and she was not jaundiced or pale. But there were tenderness and rebound tenderness in the right sided abdomen. The hemoglobin level was slightly decreased to $9.9 \mathrm{~g} / \mathrm{dL}$ and coagulation tests and platelets were normal. Laboratory data including liver function test were within normal limits except a slightly elevated serum glucose level. Tumor markers were not tested.

On a transabdominal ultrasound examination, various sized hyperechoic masses were noted in the liver. And small amount of fluid was noted in the perihepatic space and pelvic cavity. The initial differential diagnosis of liver lesions included hemangiomas and focal nodular hyperplasia $(\mathrm{FNH}) \mathrm{s}$. The appendix was normally demonstrated.

About 17 hours after the initial visit, emergency cesarean section was decided due to fetal distress. Intraoperatively, three bleeding foci were presented on the surface of ruptured liver masses and bleeder ligation was performed after the baby out. A dynamic abdominal computed tomography (CT) was performed a day after the delivery. The CT scan showed multiple solid masses in both lobes of the liver. Relatively large masses which were located in the right lobe included highly attenuated portions suggesting hemorrhage on the precontrast phase (Fig. 1A). On the arterial and portal phases, they showed intratumoral contrast leakage (Fig. 1B). There were numerous smaller masses with similar morphologic features in both lobes of liver. A magnetic resonance imaging was performed 3 days after the CT examination with a clinical 3.0-T unit (Signa HDx; GE Healthcare, Waukesha, WI, USA). It revealed multiple high signal intensity (SI) masses on T2-wighted image (Fig. 1C). Masses contained dark SI portions suggesting hemorrhage. T1-weighted images showed that the mass in the right lobe has a central, concentric rim of high SI surrounding an area of low
Correspondence to: Hae Kyung Lee

Department of Radiology, Soonchunhyang University Bucheon Hospital, 170 Jomaru-ro, Wonmi-gu, Bucheon 14584, Korea

Tel: +82-32-621-5851, Fax: +82-32-621-5874, E-mail: radhklee@naver.com

Received: Nov. 9, 2016 / Accepted after revision: Dec. 1, 2016
(C) 2016 Soonchunhyang Medical Research Institute This is an Open Access article distributed under the terms of the Creative Commons Attribution Non-Commercial License (http://creativecommons.org/licenses/by-nc/4.0/). 

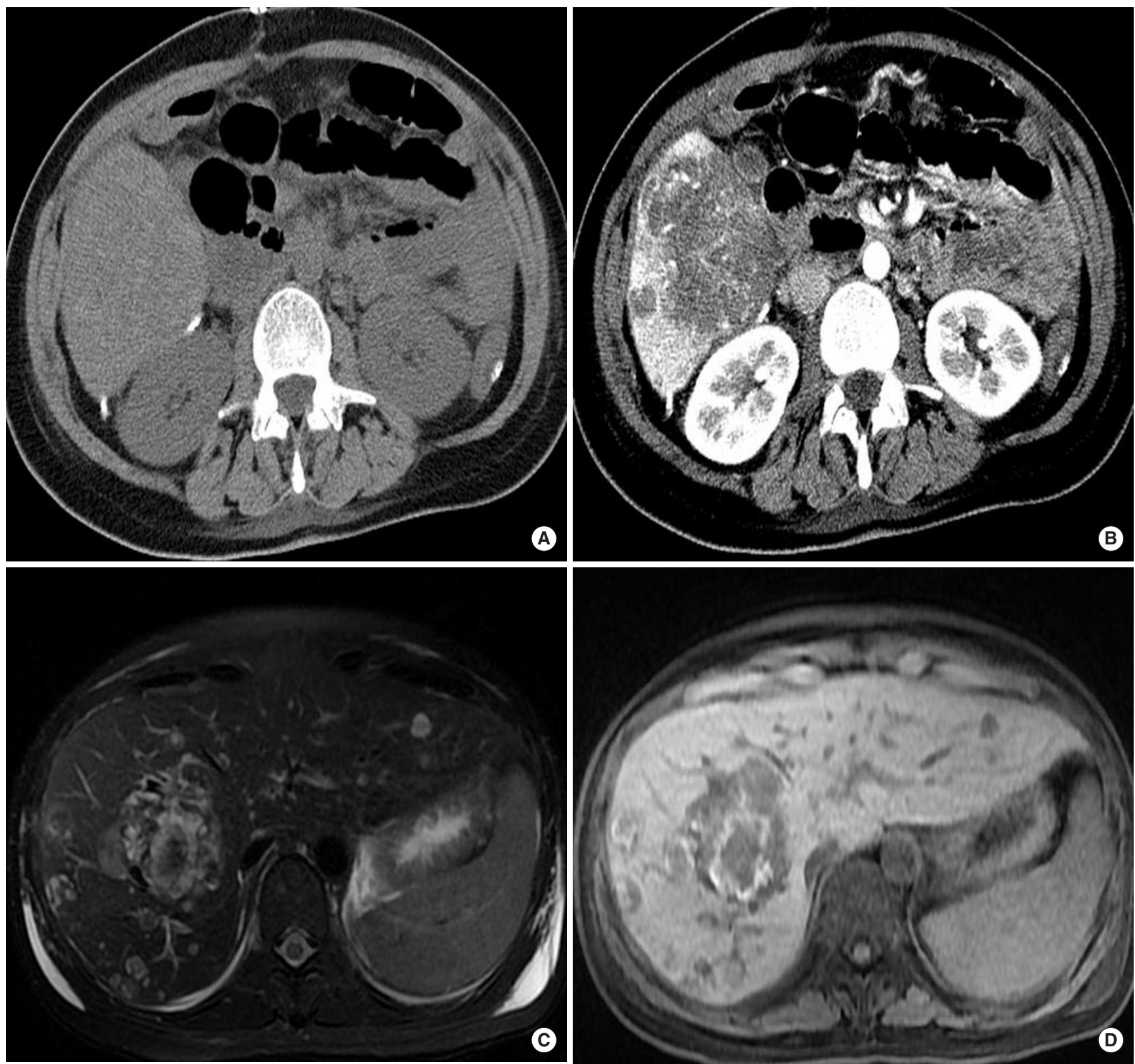

Fig. 1. 32-year-old woman with spontaneously ruptured intrahepatic cholangiocarcinoma. (A) Computed tomography images which was performed a day after the delivery showed masses with highly attenuated portions suggesting hemorrhage on the precontrast phase. (B) On the arterial phase, intratumoral contrast leakage was noted. (C) On T2-wighted magnetic resonance imaging image, multiple high SI masses contained dark SI portions suggesting hemorrhage. (D) T1-weighted image showed that the mass in the right lobe has a central, concentric rim of high SI surrounding an area of low SI. SI, signal intensity.

SI (Fig. 1D). On diffusion-weighted images, high SI foci were noted in almost all of the masses. The working diagnosis based on the images was a spontaneous hemorrhage of malignant hepatic lesions, whose differential diagnoses included angiosarcoma or metastasis. On positron emission tomography-CT images, the hepatic masses showed increased 18F-fluorodeoxyglucose uptake and there were multiple small hypermetabolic lesions suggesting me- tastasis in the both lungs and spines.

Meanwhile the patient's conditions got worse, so she underwent an emergency laparotomy to control her bleeding and a right hemi-hepatectomy was carried out. Grossly, the resected specimen of the liver demonstrated several sponge-like masses filled with blood (Fig. 2A). Microscopically, the tumors were poorly differentiated carcinoma. The results of immunohistochemistry showed a 

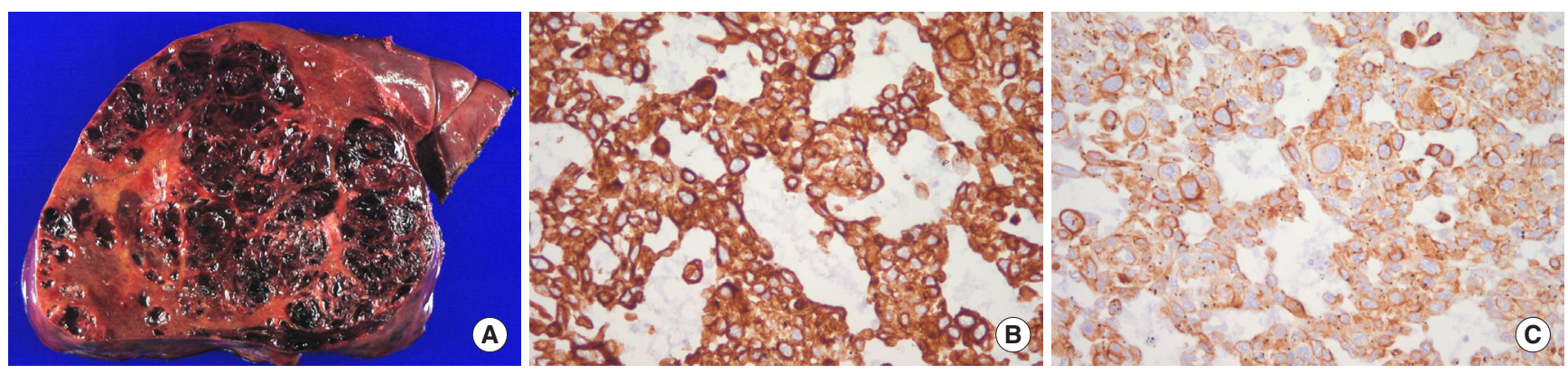

Fig. 2. (A) Resected specimen of the liver. The cut surface shows several sponge-like masses filled with blood. (B, C) Immunohistochemical results for (B) CK7 and (C) CK19 showing diffuse positive reactions of tumor cells $(\times 400)$. CK, cytokeratin.

diffuse immunoreactivity of tumor cells for cytokeratin (CK) 7 and CK 19 (Fig. 2B, C), and the absence of immunostaining for hepatocyte. On the basis of imaging and pathological features, the diagnosis of intrahepatic cholangiocarcinoma with intrahepatic metastasis was performed. In spite of the right hemi-hepatectomy, intractable bleeding from the remnant tumors and subsequent multi-organ failure was developed and the patient passed away 6 days after the surgery.

\section{DISCUSSION}

Spontaneous rupture of a hepatic tumor is a life threatening condition. The most common causes of non-traumatic hepatic hemorrhage are HCC and hepatocellular adenoma. Other tumors including $\mathrm{FNH}$, hemangioma, and metastases may rupture [1,2]. Marini et al. [3] described 20 patients with spontaneous rupture of a liver tumor associated with hemoperitoneum and the causes were HCC in 13 cases and hepatocellular adenoma in 7 cases. In another study of Chen et al. [1], the causes of 70 cases of hepatic rupture were HCC (85.7\%), hepatocellular adenoma (5.7\%), liver cirrhosis (4.3\%), hepatic hemangioma (2.9\%), and metastasis (1.4\%). In areas of Asia and Africa, rupture of HCC is reported to occur in $6.9 \%-14 \%$ of cases [2].

In contrast with HCC, cholangiocarcinoma usually forms a hard tumor with abundant fibrous stroma, so this tumor seldom ruptures spontaneously [4]. Only a few cases of spontaneous rupture of cholangiocarcinoma were reported [4-6]. Chong et al. [6] suggested a papillary type of tumor with little fibrous stroma and a capsular location as the predisposing factors to rupture of cholangiocarcinoma. Akatsu et al. [4] proposed a presence of venous obstruction as another risk factor. In a report of Ka et al. [7] these predisposing factors were also noted. But in our patient, there was no definite predisposing factor to rupture of tumor on histopathologic finding. Only her pregnancy was an unusual status.

Limited data is available regarding development of cholangiocarcinoma in pregnancy. But generally, pregnancy makes estrogen levels higher and it can theoretically aggravate a preexisting malignancy. Suppression of the immune system is another possible cause for the aggravation of the malignancy during pregnancy [8]. In our case, the symptom of bleeding was developed during the patient's pregnancy and at that time, her coagulation profile and platelets were normal. Even though we didn't know whether the pregnancy had directly influenced the rupture of tumors or not, it might be a cause of aggressiveness of tumors.

Management of ruptured cholangiocarcinoma generally follows that of ruptured HCC. Transcatheter arterial embolization (TAE), surgical hemostasis, conservative treatment, and open surgery can be considered as immediate treatment [7]. In our case, there was difficulty in making a diagnosis based solely on imaging before her delivery. In spite of surgical hemostasis, her bleeding was continued and there was no change to do TAE.

We report a rare case of a spontaneous rupture of cholangiocarcinoma in a pregnant woman. The tumor had no known predisposing factor to rupture of cholangiocarcinoma. We may consider a pregnancy as a potential cause of spontaneous rupture of cholangiocarcinoma in addition to a papillary type of tumor with little fibrous stroma, a capsular location, and a presence of venous obstruction.

\section{REFERENCES}

1. Chen ZY, Qi QH, Dong ZL. Etiology and management of hemmorrhage in spontaneous liver rupture: a report of 70 cases. World J Gastroenterol 2002;8:1063-6.

2. Casillas VJ, Amendola MA, Gascue A, Pinnar N, Levi JU, Perez JM. Im- 
aging of nontraumatic hemorrhagic hepatic lesions. Radiographics 2000; 20:367-78

3. Marini P, Vilgrain V, Belghiti J. Management of spontaneous rupture of liver tumours. Dig Surg 2002;19:109-13.

4. Akatsu T, Ueda M, Shimazu M, Kawachi S, Tanabe M, Aiura K, et al. Spontaneous rupture of a mass-forming type peripheral cholangiocarcinoma. J Gastroenterol Hepatol 2005;20:162-3.

5. Watanabe M, Akagi S, Hamamoto S. Intraperitoneal rupture of cholangiocarcinoma in a patient with liver cirrhosis, type $\mathrm{C}$, with hepatocellular carcinoma. Am J Gastroenterol 1999;94:2320-1.

6. Chong RW, Chung AY, Chew IW, Lee VK. Ruptured peripheral cholangiocarcinoma with hemoperitoneum. Dig Dis Sci 2006;51:874-6.

7. Ka SY, Leung JL, Chu CY, Wan AY, Tang VW. Intrahepatic cholangiocarcinoma presenting with spontaneous rupture. J Hong Kong Col Radiol 2010;13:91-5.

8. Marasinghe JP, Karunananda SA, Angulo P. Cholangiocarcinoma in pregnancy: a case report. J Obstet Gynaecol Res 2008;34(4 Pt 2):635-7. 